\title{
Tuberculosis management in India during COVID-19 crisis
}

\author{
Avijit Kumar Awasthi ${ }^{1}$ D Pravin Kumar Singh ${ }^{2}$
}

Accepted: 25 October 2020 / Published online: 24 November 2020

(C) Springer Nature Limited 2021

\begin{abstract}
During the early months of the 2020 COVID-19 crisis, critical services in India for tuberculosis (TB) have been disrupted. India has one of the highest burdens of TB and requires continuity of critical TB care. Here, we highlight important points and approaches that can be used to guide navigation of the combined COVID-19 and TB crisis in India.
\end{abstract}

Keywords COVID-19 $\cdot$ TB care $\cdot$ TB health workers

In 1993, the World Health Organization (WHO) declared Tuberculosis (TB) a public health emergency and called for all nations to prioritize TB control efforts. TB may be treated successfully with a standard 6-month drug-regimen. However, multi-drug resistant-TB (MDR-TB) and extensive drug resistant-TB (XDR-TB) are very difficult to treat and require longer, more complex courses of treatment. TB affects India badly with the highest incidence (2.7 million) and number of deaths ( 0.4 million) recorded worldwide in 2018 [1].The rapid and unexpected surge in demand for diagnosis, treatment, and care of COVID-19 patients has interfered with routine health services around the world and in India. Importantly, this disruption has affected important TB notification and scheduled treatments.

Although the number of COVID-19 cases has risen sharply in India, the case fatality ratio (CFR) remains low (2.8), especially in comparison to France, Italy, and the United Kingdom $(15.3 \%, 14.4 \%$, and, $14.2 \%$ respectively) [2]. The low CFR in India could be due to the timely response by the Government of India that included nation-wide lockdown, strengthening of existing health infrastructure, administrative commitment, awareness among population, dietary support and socio-economic protection for poor [3].

Avijit Kumar Awasthi

akaa21@gmail.com

1 Intermediate Reference Laboratory, State TB Demonstration and Training Centre, Agra, Uttar Pradesh, India

2 Department of Biotechnology, GLA University, Mathura, Uttar Pradesh, India 
At present, the attention of India, and the world, is focused on halting the spread of COVID-19. However, resultant gaps in TB care services could increase COVID19 case numbers as TB may increase susceptibility to SARS-CoV-2 [4] while contributing to an increase in TB related morbidity and mortality from the halt in treatment.

In India, where two deaths occur every three minutes from TB [1], real success in COVID-19 control cannot be ensured without the continuity of TB services in parallel. TB diagnosis, treatment and care in India is provided free of cost in the public sector by the Government's National TB Elimination Program (NTEP). However, a large proportion of patients seek care through private sector health providers which is largely unorganized in India. Below, we highlight key adaptation strategies for TB screening and diagnosis, treatment, and prevention that could be integrated with the COVID-19 response to maintain the momentum towards the goal of TB elimination in India.

\section{What could be done for screening and diagnosis?}

\section{Prioritize TB care as 'essential health services' during the ongoing COVID-19 crisis}

Over the last few years, India has scaled up TB health resources impressively with the goal of TB elimination in India. The COVID-19 crisis, however, has caused the diversion of vital TB health resources. Good co-ordination between TB and COVID19 response (at administrative, laboratory, treatment facility, and in the field) can be built strategically to ensure continuity of TB care services in tandem with COVID19 response. While the public health sector is at forefront, mobilization of the private health sector and joining with non-government health organizations can play an important role in mitigating the current challenges.

\section{Conduct active screening for both TB and COVID-19 simultaneously, especially in areas mapped as 'TB hotspots'}

TB and COVID-19 both affect the respiratory system and can infect the same vulnerable population. Testing for TB and COVID-19 simultaneously is, therefore, especially important in the 'TB hotspots' that are already well-mapped to the district level. It is necessary to pay special attention to slum dwellers in areas where 'social distancing' is almost impossible; transmission threat for both TB and COVID-19 might be high.

\section{Ensure 'up-skilling', motivation, and safety of healthcare workers}

Frontline health workers can be trained to distinguish between TB and COVID-19 symptoms and triage patients for appropriate downstream evaluation and treatment. TB health care workers engaged in NTEP have a basic understanding of air-borne infection control and have expanded their services close to patients' homes. Thus, 
they can contribute substantially to screening, contact tracing, and prevention with minimum additional training required. They can also raise awareness about COVID19 while continuing in efforts in TB care and prevention. State and local administrations should make efforts to expand the health work force by involving non-government organizations, encouraging community health workers, volunteers, and civil society to participate in reaching those at risk or those affected by TB or COVID-19, or both. While engaging health care workers, local administrations should ensure appropriate training, motivation, and adequate supply of personal protective equipment (PPE) for all health workers who come into contact with Severe Acute Respiratory infections (SARI), Influenza like illness (ILI) and patients with other related conditions. It is crucial to ensure that health care workers be protected as they respond simultaneously to both public health challenges.

\section{Bolster testing capacity for TB and COVID-19}

With recent approval of COVID-19 testing by the GeneXpert and TrueNAT machine [5], several TB laboratories are now engaged in COVID testing rather than TB testing. We expect the referral of TB specimens to decline sharply due to the major shift in attention to COVID-19. Thus, India must increase testing to minimize the impact on TB control program capacity (by arranging additional machines or increasing the number of shifts for laboratory testing). The Government should encourage engagement from private sector laboratories to contribute for COVID-19 testing. Furthermore, the Government's TB control program should offer upfront TB testing by GeneXpert or TrueNAT (in place of smear microscopy)for all SARI, ILI, presumptive TB and patients with related-illness in order to facilitate early initiation of appropriate treatment and minimize cross-infection.

\section{What could be done for treatment?}

\section{Ensure uninterrupted supply of TB drugs}

All peripheral public hospitals (such as Community and Primary Health Centres) need to maintained stocks of TB medicines. Community health care workers and treatment supporters should deliver TB medicines in sufficient quantities to patients' homes. These health workers may also advise the patients about tele-consultation with a treatment supervisor, in case of any complications.

\section{Increase the use of the digital platform for monitoring treatment adherence}

The extended period of lockdown has led many low wageworkers to migrate to their native homelands. To ensure that this migration does not adversely affect TB treatment, India's National Tuberculosis Elimination Programme (NTEP) should strategically track all TB patients across the country using available web-based tools (such as 'NIKSHAY', a patient management system application for eradication and 
control of TB) [1]. NTEP has introduced, at pilot scale, Information and Communication Technology (ICT) based monitoring of treatment adherence tools (99DOTS, MERM, among others) [1]. It is also time to expand these ICT-based treatment adherence supports and other digital platforms in NTEP to make medical care accessible in outreach areas. TB-contact screening also plays a critical role and should be continued.

\section{Minimize opportunities for disease transmission in hospitals}

Health care providers should develop systems to segregate patients with respiratory illnesses away from those with other illnesses. Providers can do so by means of pre-visit telephone evaluations or gathering information in another way, along with providing education and communication (IEC) materials .Health facilities should ensure appropriate infection control measures, fast track services with separate areas for COVID-19 and TB patients. Health care providers should exclude those with concomitant SARS-Cov-2 infection from mingling with on-treatment TB patients who experienced sudden recurrence of TB symptoms, worsening of health, or lack of response to treatment.

\section{What could be done to improve prevention?}

The public is increasingly concerned about infectious disease control during the ongoing COVID-19 pandemic. Because preventive measures for COVID-19 are the same as those needed for TB, it is important for government and other health public health organizations to maintain the people's awareness of proper hygiene, social distancing, and cough etiquette (measures to keep droplets from reaching others). These precautions will help to curb the spread of COVID-19, as well as the spread of TB. Sustaining awareness of the importance of these actions in the post-COVID-19 crisis period will require adequate investment by the government, forward-looking policies, and support from media and civil society organizations.

\section{Conclusion}

TB elimination efforts in India have gained the highest momentum in recent years and success will require that this momentum continue. Good co-ordination across health departments of the central and state governments, partner health agencies including non-government organizations and representatives of the private health sector will be needed to formulate and implement an integrated response for COVID-19 and TB simultaneously. Success in managing the health of the public in India depends upon the adoption of the approaches we have presented-all of demonstrated effectiveness. Only by the use of these measures can we improve the state of public health in India and around the world. 


\section{Compliance with ethical standards}

Conflict of interest On behalf of all authors, the corresponding author states that there is no conflict of interest. The views expressed here are those of the authors and not of any organizations.

\section{References}

1. Revised National Tuberculosis Control Programme. Annual status report 2019. Central TB Division, Ministry of Health and Family Welfare, Govt. of India, New Delhi, India: RNTCP. https://tbcin dia.gov.in/WriteReadData/India\%20TB\%20Report\%202019.pdf (2019). Accessed 23 Apr 2020

2. John Hopkins University and Medicine. Coronavirus Resource Centre. https://coronavirus.jhu.edu/ data/mortality (2020). Accessed 10 Jul 2020.

3. World Health Organization. India Situation Report-09 (28 March 2020) and India Situation Report-11 (12 April 2020), Coronavirus Disease (COVID-19). https://www.who.int/india/emerg encies/coronavirus-disease-(covid-19)/india-situation-report (2020). Accessed 10 Jul 2020

4. Liu Y, Bi L, Chen Y, Wang Y, Fleming J, Yu Y, Gu Y, Liu C, Fan L, Wang X, Cheng M. Active or latent tuberculosis increases susceptibility to COVID-19 and disease severity. medRxiv. 2020. https ://doi.org/10.1101/2020.03.10.20033795.

5. Indian Council of Medical Research (ICMR). Advisory: Newer Additional Strategies for COVID-19 Testing. ICMR, Ministry of Health and Family Welfare, Govt. of India, New Delhi, India. https:// www.icmr.gov.in/pdf/covid/strategy/New_additional_Advisory_23062020_3.pdf (2020). Accessed 10 Jul 2020

Publisher's Note Springer Nature remains neutral with regard to jurisdictional claims in published maps and institutional affiliations.

Avijit Kumar Awasthi M.Sc. Ph.D., is Consultant Microbiologist at State TB Demonstration Centre, Agra, Uttar Pradesh, India.

Pravin Kumar Singh M.Sc. Ph.D., is Assistant Professor at GLA University, Mathura, Uttar Pradesh, India. 